\title{
Galloping Control for Iced Conductors using Tuned Mass Dampers with time-delayed feedbacks
}

\author{
Guo $\mathrm{Li}^{1, \mathrm{a}}$, Li Li, b and Peng Zhü, c \\ ${ }^{1}$ School of Civil Engineering \& Mechanics, Huazhong University of Science \& Technology, \\ Wuhan, 430074, China \\ ali_guo@hust.edu.cn, ${ }^{\mathrm{b}}$ lili2431@163.com, czhu_peng@hust.edu.cn
}

Keywords: Galloping, Iced conductor, Time-delayed feedback, Tuned Mass Damper.

\begin{abstract}
This paper investigates the effects of time-delayed feedbacks on Tuned Mass Dampers (TMD) in the galloping control based on a theoretical model. The Runge-Kutta integration algorithm method is used to solve the model. Then we analyse the effects of the time delay $\mathrm{T}$ and the gain strength K of the time-delayed feedbacks on TMD's control effect. With time delay T increasing, the TMD's effect may be either amplified or suppressed, depending on the value of time delay chosen, which is periodic on the parameter T. For a range of values of $\mathrm{T}$ and $\mathrm{K}$, the galloping amplitude of the system with TMD and time-delay feedbacks (TTMD) is found to be lower than that of the system with TMD. The TMD with time-delayed feedbacks choosing a suitable time delay $\mathrm{T}$ and gain strength $\mathrm{K}$ may have better effects on preventing galloping of iced conductors.
\end{abstract}

\section{Introduction}

Galloping is a self-excited instability related to slender structures with non-circular cross-sections. Appearing with low frequency and large amplitude, galloping of transmission lines may cause electric accident, e.g., flashover, short circuit, line break and tower collapse. Since galloping was observed in the 1930s, extensive researches have been carried around this issue, and many passive and active devices have been proposed to suppress galloping, such as spacer, detuning pendulum, spoiler and tune mass damper (TMD) [1-6].

Among them, the TMD has received particular attention, which prevents galloping by increasing damping the energy dissipating of system. The TMD is widely used in vibration control of building, bridge and mechanical system. Previous studies are mainly devoted to design the spring-viscous connection of the added mass to the system for reaching a suitable tuning to the frequency of an assigned mode [7-11]. However, the TMD with time-delayed feedbacks may be another way to increase the energy dissipating of system and also has a better effect. In fact, time delays arising may inevitably exist [12], and time delays plays an important role in vibration control [13]. Mehmood et al. [14] studied the effects of linear and nonlinear velocity feedbacks on vibration amplitude of a circular cylinder. Abdelkefi and Ghommem [15], L. Wang et al. [16] studied the effects of time-delayed feedbacks on aeroelastic galloping response of square prisms and find that galloping responses can be either amplified or suppressed by time-delay feedbacks, depending on the time delay chosen. Besides, time-delay feedback control has been widely adopted to control the vibration of lathe cutting tools and container cranes [17, 18]. Although the effects of delayed feedback control on nonlinear vibration absorber system has been studied by $\mathrm{Xu}$ Jian [19], the galloping of transmission lines which is self-excited instability is different from that. So it is necessary to study the effects of time-delayed feedbacks on the TMD's effects in galloping control.

This paper thus focuses on the effects of time-delayed feedbacks on TMD's effects in the galloping control. The remaining parts are organized as follows: the mechanical models approximating the vibration of the system including time-delay feedbacks is presented in section 2. Section 3 gives numerical results that is, the curves of the variation of galloping amplitude of the different systems with respect to the time delay $\mathrm{T}$ and the gain strength $\mathrm{K}$. Finally, the results are discussed and summarized in section 4 . 


\section{Equations of Motion}

Simplified Model. Galloping of iced conductors can be simplified to the model shown in Fig. 1(a): a spring-mounted cylinder body of mass $m_{1}$ immersed in an incoming flow. Galloping of iced conductors-TMD system can be simplified to the model shown in Fig. 1(b): a spring-mounted cylinder body of mass $m_{1}$ immersed in an incoming flow and a secondary mass $m_{2}$ elastically connected to the galloping body $m_{1}$ which is supposed to not affected by the aforementioned flow. Similarly, galloping of iced conductors-TTMD system can be simplified to the model shown in Fig. 1(c): a spring-mounted cylinder body of mass $m_{1}$ immersed in an incoming flow and a secondary mass $m_{2}$ inelastically connected to the galloping body $m_{1}$ which is also not affected by the aforementioned flow.

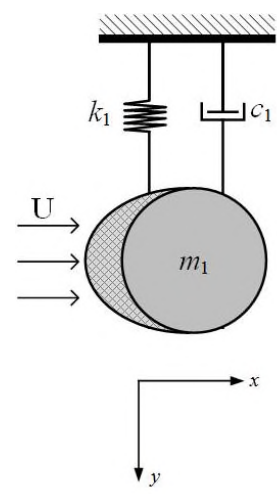

(a)

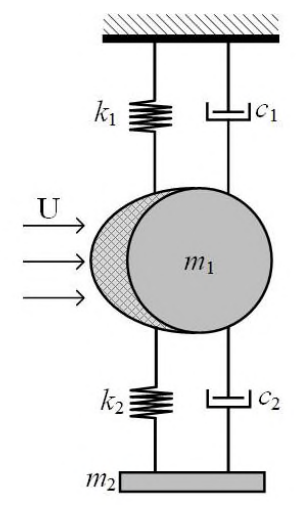

(b)

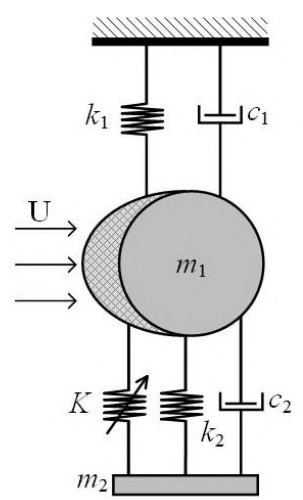

(c)

Fig. 1 Simplified model.

They are linked by linear springs $\left(k_{1}, k_{2}\right.$ and $\left.K\right)$ and damper $\left(c_{1}\right.$ and $\left.c_{2}\right) . k_{1}$ and $c_{1}$ connect the galloping body to the support, $k_{2}, c_{2}$ and $K$ connect the dual mass to the galloping body.

\section{Governing Equations}

The vertical motion of the simplified galloping model in Fig. 3(a) can be described by the following differential equation:

$$
m_{1} \ddot{y}_{1}+c_{1} \dot{y}_{1}+k_{1} y_{1}=\frac{1}{2} \rho U^{2} D C_{y}
$$

The vertical motion of the simplified galloping model in Fig. 3(b) can be described by the following differential equation:

$$
\begin{gathered}
m_{1} \ddot{y}_{1}+c_{1} \ddot{y}_{1}+k_{1} y_{1}-c_{2}\left(\dot{y}_{2}-\dot{y}_{1}\right)-k_{2}\left(y_{2}-y_{1}\right)=\frac{1}{2} \rho U^{2} D C_{y} \\
m_{2} \ddot{y}_{2}+c_{2}\left(\dot{y}_{2}-\dot{y}_{1}\right)+k_{2}\left(y_{2}-y_{1}\right)=0
\end{gathered}
$$

The vertical motion of the simplified galloping model in Fig. 3(c) can be described by the following delayed differential equation:

$$
\begin{gathered}
m_{1} \ddot{y}_{1}+c_{1} \dot{y}_{1}+k_{1} y_{1}-c_{2}\left(\dot{y}_{2}-\dot{y}_{1}\right)-k_{2}\left(y_{2}-y_{1}\right)-K\left(y_{2}(t-T)-y_{1}\right)=\frac{1}{2} \rho U^{2} D C_{y} \\
m_{2} \ddot{y}_{2}+c_{2}\left(\dot{y}_{2}-\dot{y}_{1}\right)+k_{2}\left(y_{2}-y_{1}\right)+K\left(y_{2}(t-T)-y_{1}\right)=0
\end{gathered}
$$

where, $y_{1}$ is the position of galloping body, $y_{2}$ is the position of the added mass, with respect to the zero equilibrium position of the system, $m_{1}$ is the equivalent mass of galloping body, $m_{2}$ is the equivalent mass of TMD, $k_{1}$ is the equivalent stiffness of iced conductors, $k_{2}$ is the equivalent stiffness of added mass, $c_{1}$ is the equivalent damping coefficient of iced conductors, $c_{2}$ is the equivalent damping coefficient of the TMD, $T$ is the time-delayed parameter, $K$ is the gain strength of 
time-delayed feedbacks, $f_{\mathrm{y}}$ is the vertical wind load intensity and the dot symbol stands for differentiation with respect to time $t$.

As for the aerodynamic force $f_{\mathrm{y}}$, some assumptions need to be introduced: (1) the quasi-steady theory is adopted; (2) the ice is uniformly distributed along the transmission line. Following Belvins [20], Barrero-Gil [21] and Luongo [22], a cubic polynomial is used to approximate the vertical fluid force coefficient so that the aerodynamic forces can be expressed as

$$
f_{y}=\frac{1}{2} \rho U^{2} D\left(a_{1} \frac{\dot{v}}{U}+a_{3}\left(\frac{\dot{v}}{U}\right)^{3}\right)
$$

where, $\rho$ is the air density, $U$ is the steady wind, $D$ is the diameter of the conductors, $\dot{v} / U$ is the angle of attack, $a_{1}, a_{2}$ and $a_{3}$ are the aerodynamic coefficients of the drag force, which are measured via wind tunnel tests.

\section{Numerical Results and Discussion}

In the following numerical analysis, the same parameters of the three models are selected as follows: the equivalent mass of iced conductors is $m_{1}=0.44 \mathrm{~kg}$, the equivalent stiffness of conductor structure is $k_{1}=180 \mathrm{~N} / \mathrm{m}$. the equivalent damping ratio of conductor structure is $\xi_{1}=0.515 \times 10^{-3}$, the mass of the TMD is $m_{2}=0.5 \mathrm{~m}_{1}$, the stiffness of the TMD is $k_{2}=0.5 k_{1}$, the damping coefficient of the TMD is $c_{2}=0.5 c_{1}$, the aerodynamic coefficients are $a_{1}=0.667$ and $a_{3}=-33.432$, the gravitational acceleration is $g=9.81 \mathrm{~m} . \mathrm{s}^{-2}$, the air density is $\rho=1.29 \mathrm{~kg} \cdot \mathrm{m}^{-3}$.

The solutions of Eq. (1), (2) and (3) are obtained by using Runge-Kutta integration method with variable step; the initial conditions are chosen to be $y_{1}(0)=0.01, \dot{y}_{1}(0)=0, y_{2}(0)=0, \dot{y}_{2}(0)=0$.

\section{Comparison of the Three Models}

Fig. 2 shows that the galloping amplitude varies with wind speed for different models. From Fig. 2 , we can see that the time-delayed feedbacks have effects on galloping control. For $\mathrm{T}=3 \mathrm{~s}$, the response amplitude and critical velocity of the system with TTMD are found to be lower than that of the system with TMD in the absence of time-delayed feedbacks and that of the system without TMD. The response amplitude and critical velocity of the system with TMD is lower than the single system. For $\mathrm{T}=1.5 \mathrm{~s}$, the response amplitude and critical velocity of the system with TTMD are found to be higher than that of the system with TMD in the absence of time-delayed feedbacks and that of the system without TMD. So the effects of TMD may be either amplified or suppressed by time-delayed feedbacks, depending on the value of the time delay chosen. When the appropriate time delay $\mathrm{T}$ is adopted, the effects of TMD can be dramatically amplified. The selection of the time delay $\mathrm{T}$ is analysed in the next section.

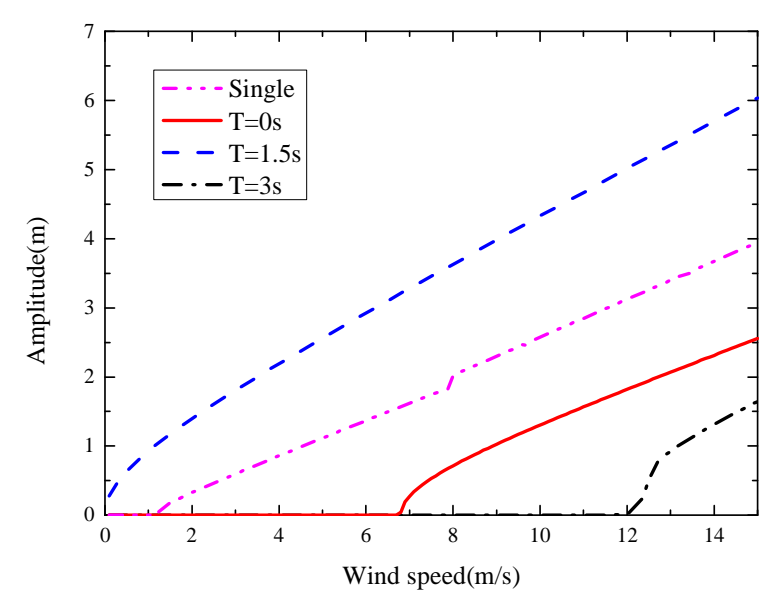

Fig. 2 Variation of galloping amplitude with wind speed for different models. 


\section{Effects of the Delay Time on Galloping Amplitude}

Fig. 3 gives the curves of the variation of galloping amplitude with time delay $\mathrm{T}$ under different gain strength $\mathrm{K}$ of time-delayed feedbacks. When $0<\mathrm{T}<2.5 \mathrm{~s}$, the response amplitude of the system with TTMD is larger than that of the system with TMD, the effects of TMD is suppressed. When $2.5 \mathrm{~s}<\mathrm{T}<3.5 \mathrm{~s}$, the galloping of the system with TTMD is prevented, which means that the effects of TMD is dramatically amplified. When $3.5 \mathrm{~s}<\mathrm{T}<4 \mathrm{~s}$, the behavior is same to that of the range from 0 to $2.5 \mathrm{~s}$. It indicates that the effect of feedback force on TMD is periodic on the time delay parameter T. In certain range, the galloping even can be prevented and the TMD's effect is markedly amplified. However, in other range, the TMD' effect can be ignored. Of course, the effects of time delayed feedbacks increase with gain strength $\mathrm{K}$ increasing.

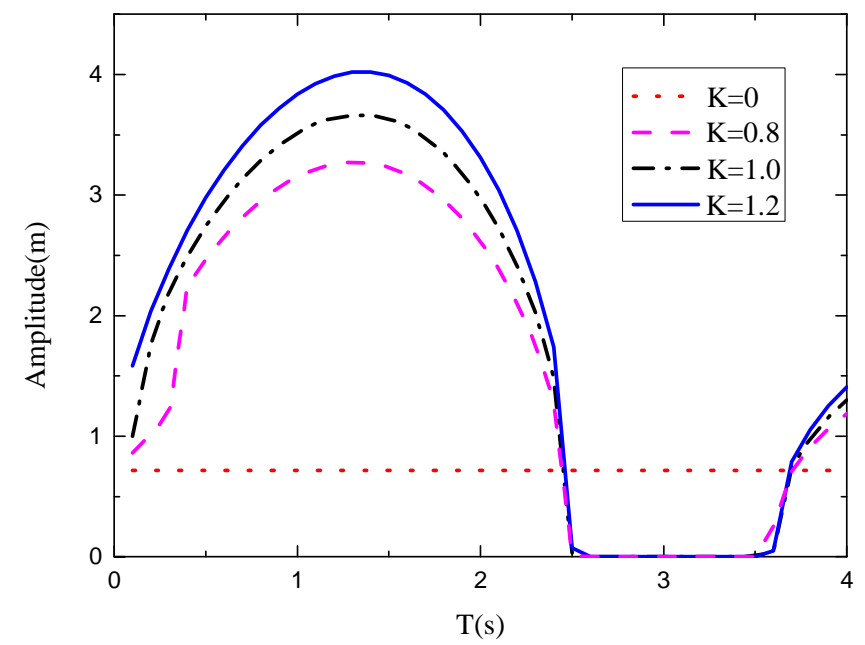

Fig. 3 Variation of galloping amplitude with delay time T under different gain strength $\mathrm{K}$ of time-delayed feedback.

\section{Effects of the Gain Strength of Feedback Force on Galloping Amplitude}

Fig. 4 presents the variation of galloping amplitude with wind speed under different gain strength $\mathrm{K}$ and delay time $\mathrm{T}$. When $\mathrm{T}=3.0 \mathrm{~s}$, the TTMD prevent galloping and the galloping amplitude decreases with gain strength $\mathrm{K}$ increasing. When $\mathrm{T}=1.5 \mathrm{~s}$, the TTMD promote galloping and the galloping amplitude increases with gain strength $\mathrm{K}$ increasing. So the increasing of the gain strength not always decrease the galloping amplitude, but always promote the effects of TMD.

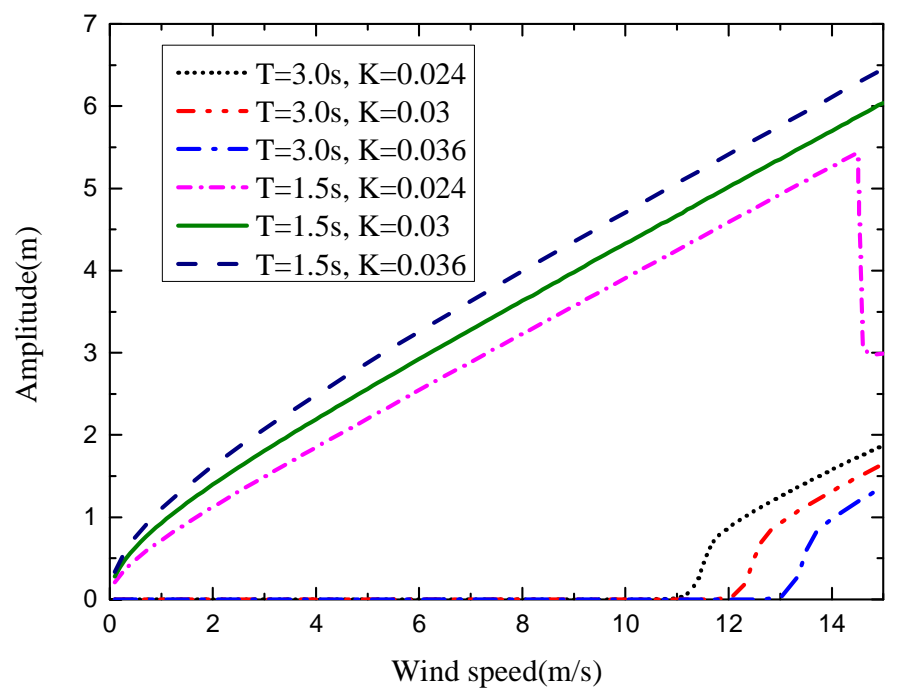

Fig. 4 Variation of galloping amplitude with wind speed under different gain strength $\mathrm{K}$ and time delay $\mathrm{T}$. 


\section{Conclusion}

In this paper, the effects of time-delayed feedbacks on Tuned Mass Dampers in the galloping control is investigated, based on a theoretical model. Three models are introduced to contrasted and solved by the Runge-Kutta integration algorithm method. Regarding the effects of the time delay $\mathrm{T}$ and the gain strength $\mathrm{K}$ of the time-delayed feedbacks on TMD, the main findings are summarised as follows:

(1) The galloping amplitude of the system with TMD and time-delayed feedbacks is periodic on the time delay $\mathrm{T}$.

(2) With the time delay T increasing, the effects of TMD may be either amplified or suppressed by the time-delayed feedbacks, depending on the value of the time delay $\mathrm{T}$ chosen.

(3) The TMD with time-delayed feedbacks may have better effects on preventing galloping.

\section{References}

[1] A. J. Carreira, Controlling Conductor Motion with Interphase Spacers in Regions of Contamination, IEEE Electric. Insul. Mag. 24(6) (2008) 35-42.

[2] M. L. Lu, N. Popplewell, A. H. Shah, J. K. Chan, Hybrid Nutation Damper for Controlling Galloping Power Lines, IEEE Trans. Power Deliv. 22(1) (2007) 450-456.

[3] M. D. Rowbottom, B. Sc., Ph.D., C. Eng, F. I. M. A., M. I. E. E., J. G. Allnutt, B. Sc., D. Phil. Mechanical dampers for the control of full span galloping oscillations, IEE PROC., 129(3) (1982) 123-135.

[4] Y. L. Guo, G. X. Li, C. Y. You, Galloping of transmission line, China Electric Power Press 2002, 9.

[5] V. Gattulli, R. Ghanem, Adaptive control of flow-induced oscillations including vortex effects, Int. J. Non-Linear Mech. 34(5) (1999) 853-868.

[6] J. L. Lilien, A. Vinogradov, Full-scale Teats of TDD Antigalloping Device (Torsional Damper and Detuner), IEEE Trans. Power Deliv. 17(2) (2002) 638-643.

[7] A. Larsen, Vortex-induced response of bridges and control by tuned mass dampers, in: Moan et al. (Eds.), Structural Dynamics_EURODYN'93, A.A. Balkema, Rotterdam, 1993, pp. 1003-1010.

[8] A. Larsen, E. Svensson, H. Andersen, Design aspects of tuned mass dampers for the Great Belt East Bridge approach span, J. Wind Eng. Ind. Aerodynamics, 54-55 (1993) 413-426.

[9] M. D. Rowbottom, The optimization of mechanical dampers to control self-excited galloping oscillations, J. Sound Vib. 75(4) (1981) 559-576.

[10]Y. Fujino, M. Ab!e, Design formulas for tuned mass dampers based on a perturbation technique, Earthq. Eng. Struct. Dynam. 22(10) (1993) 833-854.

[11]Y. Fujino, P. Warnitchai, M. Ito, Suppression of galloping of bridge tower using tuned mass damper, J. Faculty Eng., The University of Tokyo, 38(2) (1985) 49-73.

[12]Z. D. Xu, L. W. Ma, Structural Dynamics, Beijing: Science Press, 2007.

[13]A. Q. Li, Structure Vibration Control, Beijing: China Machine Press, 2007.

[14]A. Mehmood, A. Abdelkefi, I. Akhtar, A. H. Nayfeh, A. Nuhait, M. R. Hajj, Linear and nonlinear active feedback controls for vortex-induced vibrations of circular cylinders, J. Vib. Contr. 20(8) (2014) 1137-1147.

[15]H. L. Dai, A. Abdelkefi, L. Wang, W. B. Liu, Control of cross-flow-induced vibrations of square cylinders using linear and nonlinear delayed feedbacks, Nonlinear Dyn. 78(2) (2014) 907-919. 
[16]L. Wang, W. B. Liu, H. L. Dai, Aeroelastic galloping response of square prisms: The role of time-delayed feedbacks, Int. J. Eng. Sci. 78 (2014) 79-84.

[17]Z. Masoud, A. H. Nayfeh, Sway reduction on container cranes using delayed feedback controller, Nonlinear Dyn. 34(3) (2003) 347-358.

[18]Z. Masoud, A. H. Nayfeh, N. A. Nayfeh, Sway reduction on quay-side container cranes using delayed feedback controller: simulations and experiments, J. Vib. Contr. 11(8) (2005) 1103-1122.

[19]Y. Y. Zhao, J. Xu, Effects of delayed feedback control on nonlinear vibration absorber system, J. Sound Vib. 308(1) (2007) 212-230.

[20]R. D. Blevins, Flow-Induced Vibrations, Van Nostrand Reinhold, New York (1990).

[21]A. Barrero-Gil, G. Alongso, A. Sanz-Andres, Energy harvesting from transverse galloping, J. Sound Vib, 329(14) (2010) 2873-2883.

[22]A. Luongo, D. Zulli, G. Piccardo, On the effect of twist angle on nonlinear galloping of suspended cables, Comput. Struct. 87(15) (2009) 1003-1014. 